\title{
Coherent Anti-Stokes Raman Spectroscopic Thermometry in a Supersonic Combustor
}

\author{
A. D. Cutler* \\ George Washington University, Hampton, Virginia 23681 \\ P. M. Danehy ${ }^{\dagger}$ \\ NASA Langley Research Center, Hampton, Virginia 23681 \\ R. R. Springer ${ }^{\ddagger}$ \\ George Washington University, Hampton, Virginia 23681 \\ and \\ S. O’Byrne, ${ }^{\S}$ D. P. Capriotti, ${ }^{\mathbb{I}}$ and R. DeLoach** \\ NASA Langley Research Center, Hampton, Virginia 23681
}

\begin{abstract}
An experiment has been conducted to acquire data for the validation of computationalfluid dynamics codes used in the design of supersonic combustors. The flow in a supersonic combustor, consisting of a diverging duct with a single downstream-angled wall injector, is studied. Combustor entrance Mach number is 2 and enthalpy nominally corresponds to Mach 7 flight. The primary measurement technique is coherent anti-Stokes Raman spectroscopy, but surface pressures and temperatures have also been acquired. Modern design of experiment techniques have been used to maximize the quality of the data set (for the given level of effort) and to minimize systematic errors. Temperature maps are obtained at several planes in the flow for a case in which the combustor is piloted by injecting fuel upstream of the main injector and one case in which it is not piloted. Boundary conditions and uncertainties are characterized.
\end{abstract}

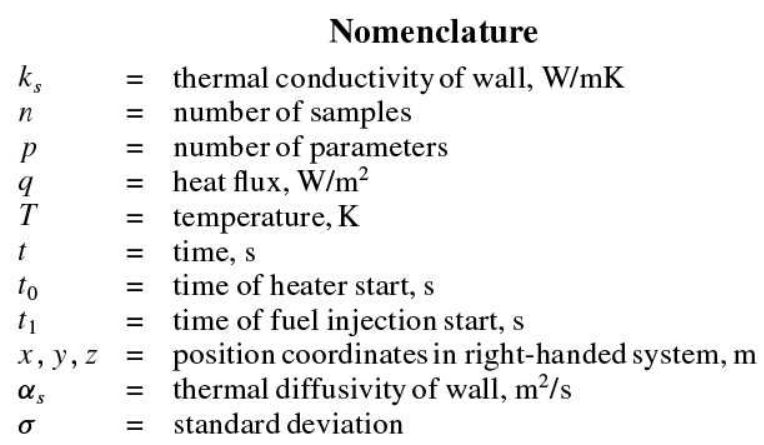

\section{Introduction}

$\mathbf{C}$ OMPUTATIONAL fluid dynamics (CFD) codes are extensively employed in the design of high-speed airbreathing engines. CFD analysis based on the Reynolds-averagedNavier-Stokes equations uses models for the turbulent momentum, energy, and mass fluxes that employ many ad hoc assumptions and empirical

Presented as Paper 2002-0743 at the Aerospace Science Meeting, Reno, NV, 14-17 January 2002; received 6 September 2003; revision received 24 September 2003; accepted for publication 30 September 2003. This material is declared a work of the U.S. Government and is not subject to copyright protection in the United States. Copies of this paper may be made for personal or internal use, on condition that the copier pay the $\$ 10.00$ per-copy fee to the Copyright Clearance Center, Inc., 222 Rosewood Drive, Danvers, MA 01923; include the code 0001-1452/03 \$10.00 in correspondence with the CCC.

*Associate Professor, MS 335. Senior Member AIAA.

${ }^{\dagger}$ Research Scientist, Instrumentation Systems Development Branch, MS 236. Member AIAA.

${ }^{\ddagger}$ Graduate Research Scholar Assistant; currently Engineering Specialist, Aerojet, Department 5272, P.O. Box 13222, Sacramento, CA 95813. Member AIAA.

$\S$ Postdoctoral Fellow, National Research Council, MS 168. Member AIAA.

IIResearch Engineer, Hypersonic Airbreathing Propulsion Branch, MS 168.

** Senior Research Scientist, Instrumentation Systems Development Branch, MS 236. Member AIAA. coefficients. Typically, these models cannot be applied with confidence to a class of flow for which they have not been validated. This experiment is one of a pair of experimentsconducted at NASA Langley Research Center that have been adopted by NATO Research and Technology Organization working group 10 (scramjets) as a test case for their CFD developmentand validation activity. Much of the material reported herein, and associated CFD work, is reported in the literature, ${ }^{1-4}$ as well as details of the other experiment, a study of a supersonic coaxial jet. ${ }^{5,6}$

The experiment was designed to provide a relatively simple case for CFD codes involving supersonicfuel injection, mixing, and combustion in a duct. The model geometry is simple, and large regions of subsonic/recirculating flow are avoided, although the model contains a small step ahead of injection to assist with flameholding. The flow at the exit of the facility nozzle that provides the test gas (the supersonic heated air "simulant") to the inlet of the model has been studied, both previously ${ }^{7}$ and herein, including CFD calculations, pitot surveys, one-dimensional analysis and coherent antiStokes Raman spectroscopy (CARS) measurements of temperature (see the following discussion). Model wall temperature and pressure measurements were acquired. The model geometry was verified using quality assurance techniques. The enthalpy of the test gas is nominally equivalent to Mach 7 flight. It was believed, on the basis of calculations performed, ${ }^{3}$ that this was hot enough to produce mixing-limited flow, that is, one for which chemical reaction of the injectedfuel proceedsat a much greater to equilibriumrate than mixing. The advantage of a mixing-limited flow is that it is insensitive to chemical kinetics and, thus, provides a more unambiguous test of CFD turbulence models. However, it was later found that significant combustion of the fuel did not occur until a point well downstreamof injection, at which point a rapid rise in pressure and temperature was observed. The implication is that, between injection and this point of "flameholding," chemical reaction greatly lagged mixing, whereas downstream of this point the flow was indeed mixing limited.

CARS is the primary experimental technique employed in this investigation. An introduction to CARS is given by Eckbreth, ${ }^{8}$ and an application of CARS to supersonic combustors is given by Smith et al. ${ }^{9}$ In the present application, molecular nitrogen is probed to obtain temperature. CARS, like many other optical techniques, does 
not significantly alter the flow being studied. Intrusive probes, such as pitot, total temperature, hot-wire, and so forth, are not used due to access difficulty and high heat flux in the combustor, and because they may alter the flow. CARS has several advantages over other optical methods. It is relatively mature and well understood. The signal is in the form of a coherent (laser) beam and is collected through relatively small windows. Incoherent (non-CARS) interference is then rejected by spatial filtering. Consequently, signal-to-noiseratios are typically high when compared to techniques such as Rayleigh and Raman scattering. Furthermore, thermometry based on Rayleigh and Raman techniques often assumes the knowledge of pressure, which is not known in supersonic flow, ${ }^{10}$ whereas CARS is relatively insensitive to modest pressure variations. Imaging techniques such as planar laser-induced fluorescence ${ }^{11,12}$ or filtered Rayleigh scattering ${ }^{13}$ offer instantaneous, quantitativemaps of temperature in high-speed flows. However, a desirable aspect of CARS is that it is more suitable for multiparameter measurements suitable for compiling statistics about the flow. These statistical correlations can be used to developturbulencemodels for CFD. For example, the CARS system described in this paper is currently being modified to allow simultaneousmeasurementof temperatureand species mole fraction and, hence, to allow quantification of temperature-species correlations. CARS has been used to instantaneouslymeasure temperature along a line. ${ }^{14}$ However, due to the high-temperature, low-density conditions in the present experiment, we restricted measurements to a single point to maximize the signal intensity.

Application of a complicated technique like CARS in high-speed engine environments is not routine. Because it is a pointwise (rather than planar) technique, building a "picture" of the internal temperature field of the combustor requires hundreds of facility runs, which is expensive. These problems of experimental complexity and large data sets were mitigated by use of modern design of experiments (MDOE) techniques. Specifically, due to the complexity of the experiment there are likely to be significant uncontrolled variables, including those as sociated with facility operation, the model, instrumentation, and so on. ${ }^{15-17}$ Randomization techniques ${ }^{18}$ are used to minimize systematic errors associated with these variables. Surface response methods ${ }^{19-22}$ are used to represent the spatial dependence of temperature in each of a number of planes by a mathematical function of the spatial coordinates. These methods optimize the quantity of data that needs to be acquired to meet specific goals for the random error of temperature surfaces and provide a compact, functional form for these surfaces. Additional details of the application of MDOE to this experiment are described by Danehy et al. ${ }^{2}$

\section{Flow Facility}

The experiment was conducted in NASA Langley's directconnect supersonic combustion test facility (DCSCTF; information available online at http://wte.larc.nasa.gov/facilities.cfm? id=2 [cited 29 September 2003]). Hot air simulant, known as vitiated air, is produced in the "heater" shown in Fig. 1. Molecular oxygen and air are premixed and $\mathrm{H}_{2}$ is burned in this $\mathrm{O}_{2}$-enriched air. Flow rates are selected so that the mass fraction of $\mathrm{O}_{2}$ is the same as that of standard air. The high-pressure vitiated air is accelerated through a water-cooled convergent-divergent nozzle before entering the test model. The facility is designed to test the combus-

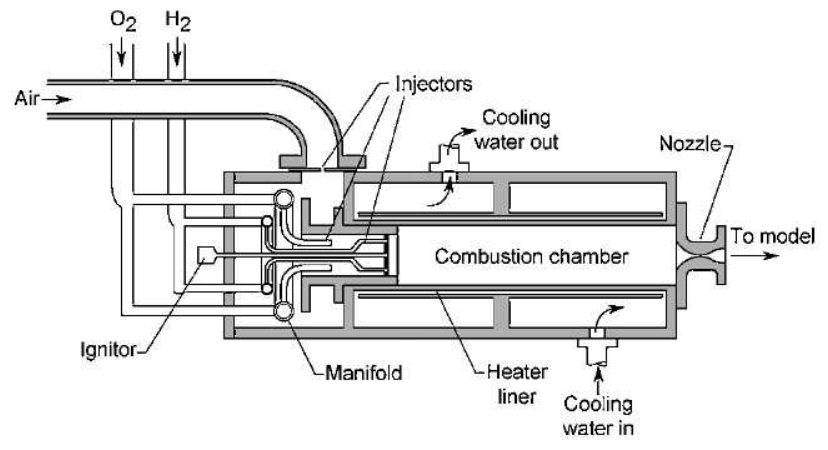

Fig. 1 DCSCTF heater and nozzle. tor of a supersonic combustion ramjet (scramjet) engine by directly connecting the facility nozzle exit to the entrance of the combustor.

The test conditions are nominally representative of Mach 7 flight. Gas flow rates to the heater are $0.915 \pm 0.008 \mathrm{~kg} / \mathrm{s}$ for air, $0.0284 \pm 0.0006 \mathrm{~kg} / \mathrm{s}$ for $\mathrm{H}_{2}$, and $0.300 \pm 0.005 \mathrm{~kg} / \mathrm{s}$ for $\mathrm{O}_{2}$. The heater stagnation pressure is $0.765 \pm 0.008 \mathrm{MPa}$. All uncertainties presented in this paper are based on the $95 \%$ probabilitylimits $(1.96$ times the standard deviation). The aforementioneduncertaintiesare due to the random run-to-run variations and do not include $\pm 3 \%$ uncertainty in the mass flow rate measurements.

Heater and nozzle exit conditions are estimated from the flow rates, heater pressure, and nozzle minimum and exit areas using an inviscid one-dimensional analysis. The flow exiting the heater into the nozzle is assumed to be in thermodynamic equilibrium but has unknown enthalpy due to heat lost to the structure and cooling water. The enthalpy is guessed and the area of the sonic throat is computed by one-dimensionalanalysis, assuming isentropic flow in the nozzle. The enthalpy is then iterated until the computed area at the sonic throat equals the geometrical minimum area of the nozzle. Nozzle exit conditions are computed from the geometrical exit area. The composition at the nozzle throat and exit could be evaluated at frozen (at heater values) or equilibrium conditions. All significant minor species are included. Calculations assuming equilibrium and frozen composition differ in minor species concentration but not significantly in major species, temperature, or pressure. The nominal calculated conditions, and uncertainties due to mass flow rate measurement error and run-to-run variations in heater conditions, are heater stagnation temperature, $1827 \pm 75 \mathrm{~K}$; exit temperature, $1187 \pm 60 \mathrm{~K}$; exit pressure, $100 \pm 1.5 \mathrm{kPa}$; and exit Mach number, $1.989 \pm 0.005$. Errors arising in the calculation due to the assumption of one-dimensional flow (the effects of nonuniform velocity, composition, temperature, etc.) are believed small.

A study of the flow quality at the exit of the facility nozzle was previously conducted? A pitot probe rake was employed to map the pitot pressure and the flowfield was visualized with the facility running as a freejet (the model removed). Silane $\left(\mathrm{SiH}_{4}\right)$ was added to the heater $\mathrm{H}_{2}$ and burned to form silica particles in the heater. The particles were illuminated by a pulsed laser-sheet placed at the nozzle exit and imaged with a charge-coupleddevice camera. Results were compared to CFD calculations of the nozzle flow. Whereas the pitot pressure varied at the nozzle exit plane, the computed pitot pressure distribution agreed well with measurement. The flow appeared qualitatively more uniform in composition following a modification to the configuration of the facility's ignitor.

The test model is shown in Fig. 2; flow direction is from left to right. The model consists of two main sections of duct: a copper upstream section and a carbon-steel downstream section. Stainless steel flanges and carbon gaskets separate the sections from each other and the nozzle. Proceeding from left to right, there is a constant-area segment, a small outward step at the top wall, and a second short constant-area segment followed by a segment with constant 3-deg divergence of the top wall. The span is constant at $87.88 \mathrm{~mm}$. Five small pilot fuel injector holes are located ahead of the step, and the main fuel injector is located just downstream of the start of the 3-deg divergence. The injection angle is $30 \mathrm{deg}$ to the opposite wall. The injector nozzle is designed by the method of characteristicsto produce Mach 2.5, one-dimensional flow at the injector exit. Molecularhydrogen injection is provided at a stagnation pressure of $2.12 \pm 0.07 \mathrm{MPa}$, a stagnation temperature of $302 \pm 4 \mathrm{~K}$, and an equivalence ratio of $0.99 \pm 0.04$. On some runs, additional $\mathrm{H}_{2}$ injection is provided by the pilot injectors at the same stagnation temperature and a total equivalence ratio of $0.148 \pm 0.008$. The pilots are turned on and off at the same time as the main fuel injector.

The duct is uncooled; however, the wall thickness of the copper duct is greater than $32 \mathrm{~mm}$ and the carbon steel duct is $19 \mathrm{~mm}$ thick. Thus, given the good thermal conductivity of these materials, it is possible to operate the facility with the model fueled for run times in excess of $20 \mathrm{~s}$ (and unfueled for much greater times) without reaching excessive temperatures. With atmospheric-temperature air flowing in the model between runs, runs could be repeated every $10-15 \mathrm{~min}$. 


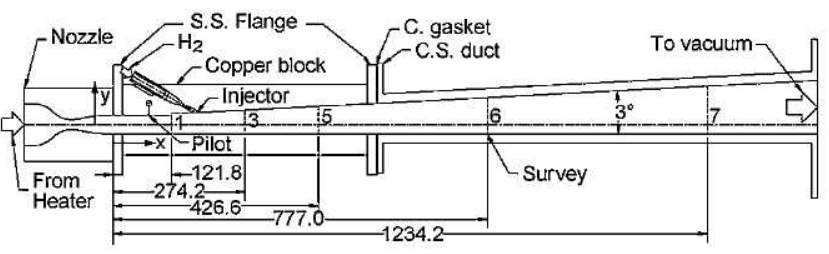

a)

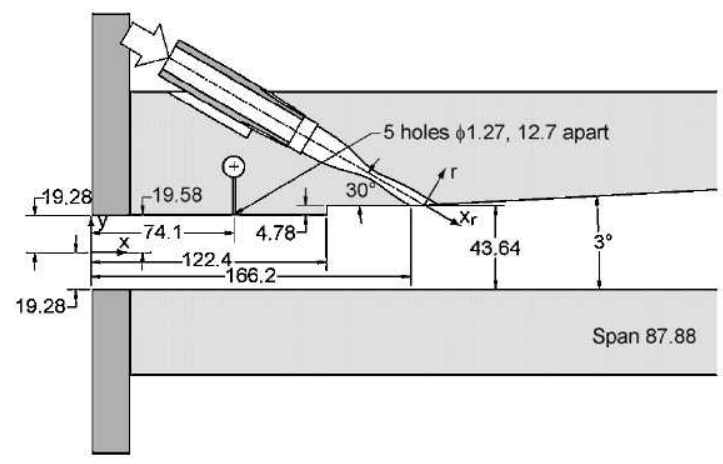

b)

Fig. 2 Test model: a) nozzle, copper and steel duct sections; b) detail in vicinity of fuel injector and pilots. Dimensions are in millimeters.

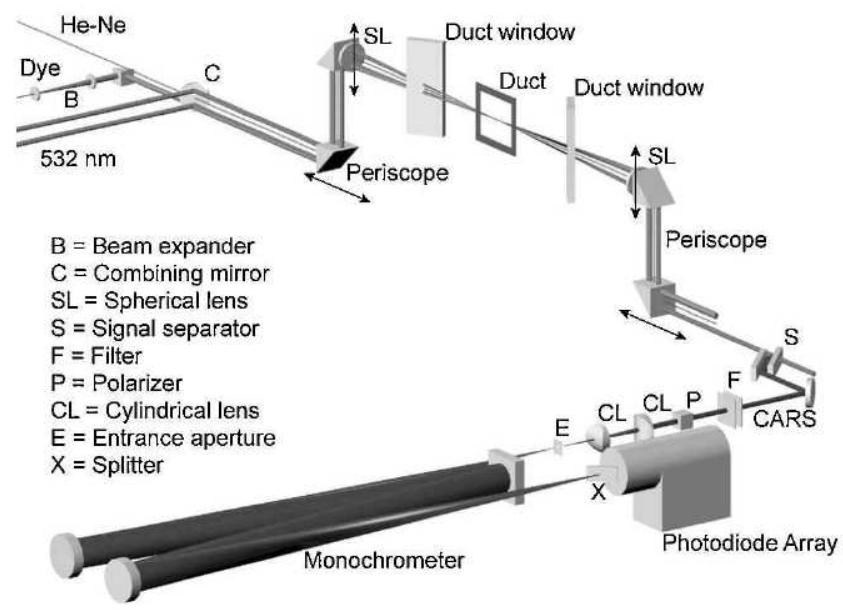

Fig. 3 CARS system: beam-combining optics; intersection and signal detection optics.

The model is equipped with seven slots to allow the CARS beams to penetrate the duct, of which slots $1,3,5,6$, and 7 , depicted in Fig. 2a, are used in this study. The slots are paired, one on each side of the duct. They are $4.8 \mathrm{~mm}$ wide and extend the full height of the duct. When not in use, the slots are plugged flush to the wall. Windows covering the slots are mounted at the end of short rectangular tubes at the Brewster angle to minimize reflections (Fig. 3). The window tubes are ventilated with a constant flow of electrically heated $(\sim 400 \mathrm{~K})$ dry air to prevent condensation of water from the heater on the windows. The CARS interaction region can thus be translated the full span and height of the duct without damaging the windows.

The model is instrumented with both pressure taps and wall temperature probes. Thirty-five static pressure taps are located in the copper duct, consisting of 0.80 -mm-diam square-edged holes. Taps are located on the bottom wall, at the centerline, and on the top wall at $z=-36.3 \mathrm{~mm}(z$ is measured from the horizontal centerline). Forty-nine static pressure taps are located in the steel duct, consisting of 1.6-mm-diam square-edged holes. Taps are located at top and bottom wall centerlines and sidewall midpoints. Pressures are measured with an accuracy of $\pm 0.6 \mathrm{kPa}$ by a Pressure Systems, Inc., electronically scanned pressure-measuring system.
Six spring-loaded, bayonet, ribbon, K-type thermocouples from Nanmac Corp. are located in 6.35-mm-diam blind holes (from the outside) in the copper block. The bottom of each of these holes is square and the wall thickness (between hole base and duct interior surface) is $2.8 \mathrm{~mm}$. A thermocouple junction is located at the bottom of each hole at the center. This choice of diameter and wall thickness is based on a multidimensional heat transfer analysis so that the thermocouple measures the unperturbed duct surface temperature. (While the temperature at the bottom of the hole is less than local duct surface temperature, the effect of the hole, which alters the conduction path, is to raise the local duct surface temperature. These effects offset.) Six "eroding" K-type thermocouples from Nanmac Corp., 7.9-mm diam and incorporatinga carbon-steel sheath, are located in the carbon-steelduct. The thermocouple junction for these probes is located flush with the duct flow surface. Over time, the junction was removed by the hot flow but was periodically regenerated by rubbing with sandpaper.

\section{CARS Technique}

\section{Optical System}

The CARS system uses a Spectra-Physics DCR-4 unseeded, pulsed Nd:YAG laser, frequency doubled to $532 \mathrm{~nm}$. The nominal power is $550 \mathrm{~mJ}$ per pulse in the green, repetition rate is $10 \mathrm{~Hz}$, and nominal linewidth is $\sim 1 \mathrm{~cm}^{-1}$. A broadband dye laser was also used, consisting of an oscillator cavity formed between a total reflecting mirror and a $30 \%$ reflector, and a single amplifier stage. Two identical Brewster-angle dye cells are used, one in the oscillator and one in the amplifier, through which flows rhodamine 640 in methanol. The wavelength is centered between 605 and $606 \mathrm{~nm}$, by adjusting the dye concentration, to match the Raman shift of nitrogen. Figure 3 shows a schematic of the remaining components of the CARS system. The dye beam is expanded in a beam expander. Dye and 532-nm beams are combined with a dichroic mirror and are relayed via a periscope to a spherical (focusing) lens of focal length $0.41 \mathrm{~m}$. All three beams cross at their focal points: the focal point of the dye beam is made coincident with the focal point of the $532-\mathrm{nm}$ beams by adjusting the beam expander. Beams are phase matched in a vertical planar BOXCARS configuration. ${ }^{8}$

At the lens, the full (to $\sim 10 \%$ of peak) diameter of the $532-\mathrm{nm}$ beams is $\sim 8.5 \mathrm{~mm}$, and the beams are $18 \mathrm{~mm}$ apart. The full diameter of the dye beam is $\sim 11 \mathrm{~mm}$. At the focus, the diameters are, respectively, $\sim 0.12$ and $\sim 0.15 \mathrm{~mm}$, yielding a geometrical beam intersection length of $5.4 \mathrm{~mm}$. The length of the measurement volume was measured by translating the CARS measurement volume through a thin planar jet of nitrogen, surrounded by a coflowing jet of helium. The length over which there was any CARS signal is $\sim 4.5 \mathrm{~mm}$ and the full-width half maximum is $\sim 2.25 \mathrm{~mm}$. The beam energy level per pulse at the focusing lens is $\sim 85 \mathrm{~mJ}$ for each of the green beams and between 12 and $24 \mathrm{~mJ}$ for the dye.

The beams (including the CARS signal beam) are relayed via a second spherical (collimating) lens and a second periscope back to the optical bench. The overlapping CARS and 532-nm beams are separated in a splitter. The splitter consists of two 100-mm-long dichroic mirrors that reflect $\sim 99.5 \%$ of the incident CARS signal, while reflecting only $\sim 20 \%$ of the $532-\mathrm{nm}$ beams and transmitting the rest. The CARS and 532-nm beams enter the splitter at 45 deg to the mirrors and undergo six back-and-forthreflections. The beam is then directed through additional filters as needed to reject residual 532- $\mathrm{nm}$ beams or for CARS signal attenuation. It enters a polarizer that allows only p-polarized light to pass (not a critical component because it is found that the signal is already well polarized). It is then focused by a pair of cylindrical lenses and enters a $1-\mathrm{m}$ monochrometer with 1200 groove/mm grating via an aperture. The cylindrical lenses are adjusted to produce a horizontal focus at the detector (maximizing image sharpness) and a loose vertical focus (to ensure the detector provides a linear response to signal ${ }^{23}$ ). An optical splitteris located in front of the detector. ${ }^{24}$ The splittercreates a secondary signal on the detector identical to the primary but offset and of $6 \%$ the intensity. When the intensity of the primary signal exceeds the dynamic range of the detector, the secondary signal is used for analysis. An EG\&G PAR model 1420 intensified, linear, 
self-scanned silicon photodiodearray detector is mounted at the exit plane of the monochrometer. It consists of 1024 elements, $2.5 \mathrm{~mm}$ high by $25 \mu \mathrm{m}$ wide, and utilizes a fast ( $\sim$ millisecond decay rate) P-46 phosphor. The detector was scanned at $30 \mathrm{~Hz}$, three times for each laser firing. The first scan was immediately after the laser pulse and acquired CARS signal, the second was used to clear the detector of any residual signal, and the third was used to determine the signal baseline.

The two top prisms of the periscope are mounted on steppingmotor-driven vertical translation stages. The two bottom prisms and the vertical translation stages are mounted on similar horizontal stages. By translating the vertical and/or horizontal stages in tandem (maintaining alignment) the measurement volume is moved in the $y$ and/or $z$ direction. It is important that the beams are parallel to the direction of motion of the translation stage. During alignments, a reference, low-power helium-neon laser $(\mathrm{He}-\mathrm{Ne})$ beam is directed through the periscopes parallel to the direction of motion of both sets of stages and through the center of the sphericallenses. The Nd:YAG and dye laser beams are periodically adjusted to ensure that they intersect the $\mathrm{He}-\mathrm{Ne}$ beam at their focus. CARS data acquisition is under the control of a personal computer. Two types of acquisition are employed. In the first, data are acquired at a single point in space. In the second, data are acquired while either the vertical or the horizontal stages are in constant-velocity motion.

CARS data are acquired in the supersonic combustor during multiple sets of test runs. During a set of runs (which might last as long as $5 \mathrm{~h}$ ), access to the model and optical system is prohibited. Test runs consisted of approximately $5 \mathrm{~s}$ during which the heater is operating but no fuel is injected in the model, followed by 11-20 s of fuel injection. CARS data are acquired over a period $2 \mathrm{~s}$ shorter than the period of fuel injection. Immediately after a run, $10 \mathrm{~s}$ of data is acquired with the dye laser beam blocked by a remotely operated flag. These "background" scans measure non-CARS interferences such as scattered laser light.

Immediately before and after a set of test runs, "reference"CARS spectra are acquired in a low-speed jet of "PC-Duster" refrigerant gas. Because this jet contains no nitrogen, and is of constant CARS susceptibility, the spectra reflect the spectral variation in dye laser power. Additionally, spectra are acquired with room-temperatureair flowing in the duct.

Large temperature variations occur in the test cell because it is continuously ventilated with external atmospheric air. Significant heat is also radiated from the model. Thermal expansion effects on the mechanical components of the optical system are not sufficient to require remote adjustments. (Adequate signal levels are typically maintained for several hours.) However, thermal expansions significantly change dye laser power and center wavelength. Because it is possible for effects such as these to generate time-varying bias errors, MDOE quality assurance tactics were used. These tactics were designed to defend against such errors, to the extent allowed by constraints in the operation of the apparatus, which will be discussed further.

\section{Data Reduction}

CARS data are analyzed on a workstation, separate from the data acquisition computer, after the data are acquired. Background scans are averaged and subtracted from data scans. Both primary and secondary (produced by the splitter) CARS signal sare contained within the data scan. If the primary signal counts exceed the linear regime of the detector, the secondary is selected for analysis. Data scans are divided by the reference spectrum to remove the effect of the dye laser spectral power distribution and normalized to unit area (primary or secondary). Data are compared to a library of similarly normalized theoretical spectra to determine the temperature and nitrogen concentration. The pixel location of the start of the theoretical spectra is allowed to float \pm 10 pixels from a nominal position in 0.3 pixel increments. The combination of temperature, concentration, and pixel location that produces the least mean square deviation between theory and data is selected.

The absolute concentration of nitrogen can be determined from spectral fitting if the optical system has been calibrated and the laser intensities are measured on a shot-by-shotbasis. ${ }^{25}$ However, for the sake of simplicity, this was not done in the current experiment. Instead, the relative concentration of nitrogen can be determined from spectral fitting if the CARS nonresonant susceptibility is known or can be reliably estimated. However, spatial variations in the composition of the test gas were not quantified in the current experiment, so the local nonresonant susceptibility could not be computed, nor could a spatially averaged value of the nonresonant susceptibility be estimated that would be within a factor of 2 of the local nonresonant susceptibility. Therefore, neither absolute nor relative nitrogen concentrationmeasurements could be determined in the present experiment. Nitrogen concentration was used as a fitting parameter in the data analysis simply to allow the spectral code to improve the quality of the fit, allowing the temperature to be determined accurately; the resulting values of nitrogen concentration were not quantitative and are not presented in this paper.

Theoretical CARS spectra are generated using the program CARSFT. ${ }^{26}$ The combustion gases are assumed to be a mixture of nitrogen and nonresonant buffer gas, both having nonresonant susceptibility of $8.5 \times 10^{-17} \mathrm{~m}^{3} / \mathrm{J}$. The nominal static pressure is assumed to be $1 \mathrm{~atm}(101,325 \mathrm{~Pa})$. In fact, as will be seen later, the pressure in the duct at the CARS measurement planes is as much as $20 \mathrm{kPa}$ above or below this value. The error introduced by this assumption is estimated by calculating theoretical spectra over a range of pressures and temperatures and fitting to them assuming $1 \mathrm{~atm}$ pressure. The differencebetween fit and true temperatures (fit minus true) is $\sim 25 \mathrm{~K}$ per $10 \mathrm{kPa}$ difference from 1 atm for $T \mathrm{~s}$ near $2000 \mathrm{~K}$. The exponential gap model for collisional narrowing of the Raman line shape is used: a Voigt model was also tried and found to make little difference for the test conditions. A 532-nm laser linewidth of $1 \mathrm{~cm}^{-1}$ is assumed, though computed temperatures are not sensitive to the 532-nm laser linewidth for the test conditions. An experimentally determined instrument probe function is used. The width of this probe function was varied for best fit to a roomtemperature CARS spectrum acquired at the beginning of each day of runs. Temperatures from 150 to $3000 \mathrm{~K}$, in increments of $25 \mathrm{~K}$, and 29 nonuniformly spaced concentrations of nitrogen are used in the library.

The peak of the reference CARS spectrum shifts significantly during a set of runs. This shift is largely due to a shift in the dye laser wavelength. Thermal effects on mechanical components of the optical system, beam steering by the hot gases in the duct, and even translation of the periscope stages all can change the position of the CARS beam focus at the entrance to the monochrometer and, hence, the position of the spectra on the detector. Two techniques are employed to correct for these problems. The first is to allow the pixel location of the start of the theoretical CARS spectra to float in the fitting process, as described. The second is to estimate the reference spectrum from the run data themselves. The technique is inspired by the observation that the directly measured dye laser spectra could be accurately fit to a Gaussian function.

The procedure is illustrated in Fig. 4. The first step is to analyze the data from a given run using the reference spectrum obtained with the refrigerant gas at the end of a set of runs. For a particular laser pulse, spectrum A is the best-fit theoreticalCARS spectrum, B is the measured CARS spectrum (prior to normalization by the reference spectrum), and $\mathrm{C}$, the ratio of $\mathrm{B}$ to $\mathrm{A}$, is an estimate of the reference spectrum for this particular shot. All such estimated reference spectra for a given run are averaged and fit to a Gaussian function for amplitude, center, and width. The region of fit is indicated in the figure and excludes a region around the bandhead of nitrogen (which tends to be noisy) and the region to the left of pixel 275 (which is affected by masking from the optical splitter). As a practical matter, it is necessary to exclude from the average individual spectra for which good fits to theory could not be obtained. Acceptable averages for fitting could be found for almost all runs. The Gaussian fit $D$ is then used as the reference spectrum for normalization of all data for the given run. The figure shows data from a laser pulse after normalization, and the best-fit theoretical spectrum. This procedure is typically iterated twice, with the latest best-fit theoretical spectrum used to determine an improved reference spectrum. 


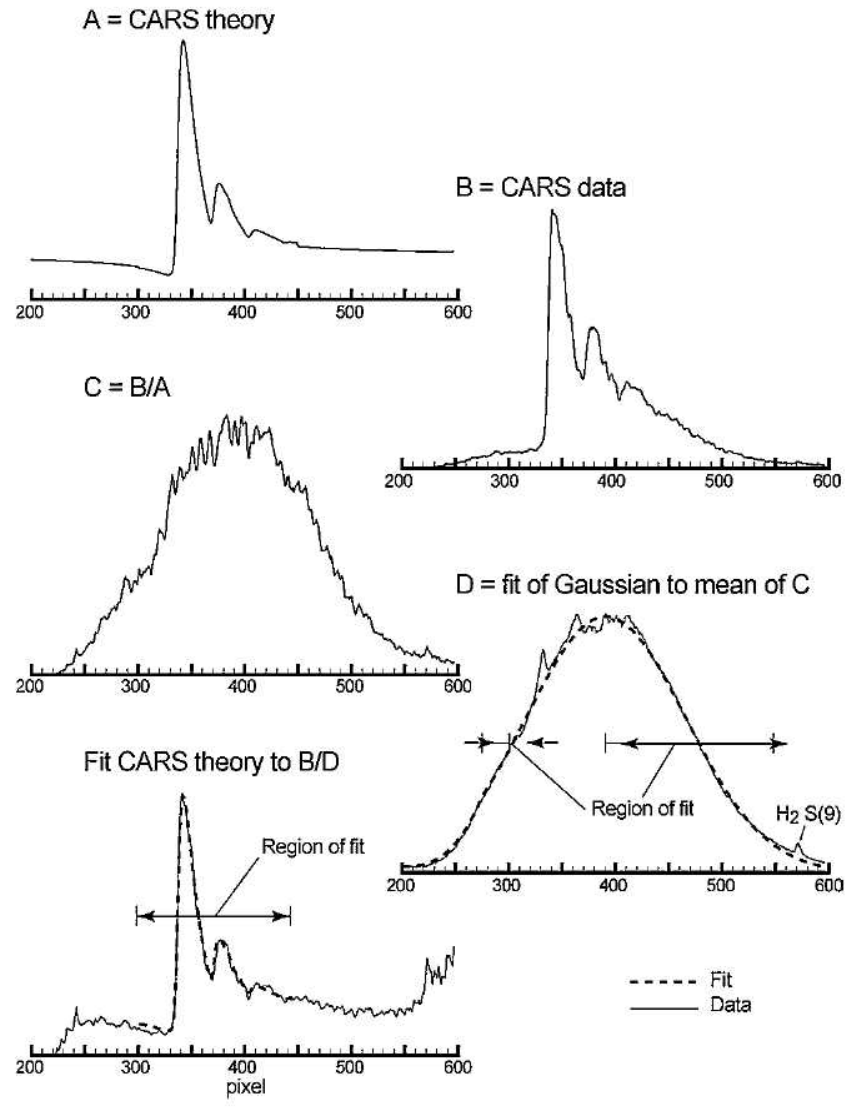

Fig. 4 CARS data analysis.

The techniques used for acquisition and analysis of CARS data in the supersonic combustor were tested in a "Hencken" adiabatic, flat-flame burner burning $\mathrm{H}_{2}$ in air. Equivalence ratio was varied and 100 laser shot data runs were performed. The mean measured temperatures were found to be within $\pm 50 \mathrm{~K}$ of temperatures calculated from measured flow rates, assuming equilibrium chemistry (including minor species). Data were also acquired in which 1) the total laser power was varied from 200 to $550 \mathrm{~mJ}$ and 2), through the use of different neutral density filters, the signal in the primary was saturated, forcing use of the secondary in analysis. No trends are found with either of these variables, indicating that the nitrogen spectrum is not saturated by high laser powers and that the splitter device works well. Standard deviations of temperature for a run are between 60 and $100 \mathrm{~K}$. The trend with increasing laser power is to lower standard deviations owing to increased signal-to-noise ratios. Thus, the uncertainty ( $95 \%$ probability) of a single-shot CARS temperature measurement is $\sim \pm 200 \mathrm{~K}$, whereas uncertainty due to errors that are fixed over a run is $\sim \pm 50 \mathrm{~K}$. A previously noted discrepancy ${ }^{1}$ in which the mean was found to be $\sim 150 \mathrm{~K}$ high at high equivalence ratio was subsequently shown to be an error due to a nonuniform flame induced by operation of the burner at $30 \mathrm{deg}$ to the vertical (because of space constraints), and not a CARS error.

\section{Design of Experiment}

It is inevitablein a complex experiment such as this that there are sources of systematic variation that are not controlled. These sources might be unidentified, or it may not be possible to correct for them. One example of a systematic variation that cannot be controlled in this experiment is the steady increase in wall temperature of the model that occurs during each run. For example, if this were to result in a steady increase in temperature throughout the flow, and if the data were all acquired in constant-velocity scans taken from left to right, then temperature maps would indicate the flow temperature in error (compared to the mean) high on the right and low on the left. Other examples include instrumentation drift, and instrument and tunnel operator fatigue and learning effects. It is possible to defend against these types of error by acquiring data in random order of spatial coordinate. This randomization has the effect of converting systematic errors into random errors. Unbiased estimates of mean temperature may then be obtained simply by averaging measurements at a point taken at different times.

Practical constraints prevent the spatial order of data points from being fully randomized.Limited stepper motor speed causes motion from one point to the next to take significant time: if it is required to move between randomly selected points during the course of a limited-time facility run, the number of data points acquired would be considerably reduced. Changing from one plane to the next requires shutdown of the facility and hardware changes. Thus, all data at a given plane are acquired in a consecutive series of runs. During a given facility run, data are acquired either at fixed points or during continuous constant-velocitymotion of the CARS measurement volume, as described in the following. However, the order of these runs and directions of the individual motions are randomized.

Data were acquiredover 201 facility runs over 10 test days. Except for one day, when laser beams clipped the edge of the duct window slots due to thermal expansion of the duct during runs, the vast majority of the data were found acceptable and were analyzed. At each plane, data were acquired at six or seven fixed points near the horizontal centerline, $6 \mathrm{~s}$ with only the heater operating followed by $18 \mathrm{~s}$ with both heater and fuel injected. Data were also acquired during $16 \mathrm{~s}$ of horizontal motion of the translation stages at $5 \mathrm{~mm} / \mathrm{s}$, or from $9 \mathrm{~s}$ to $18 \mathrm{~s}$ of 5 or $6 \mathrm{~mm} / \mathrm{s}$ vertical motion, during which time fuel was continuously injected. Two cases were considered. In the first "unpiloted" case the pilot injectors were not operated and data were acquired at planes $1,3,5,6$, and 7 . In the second "piloted" case the pilot injectors were operated and data were only acquired at planes 1, 3, and 5 (due to facility availability constraints). Figure 5 shows a three-dimensional cutaway view of the duct with the locations of the data points overlaid. Flow direction is from top left to bottom right.

Surface response techniques were employed to estimate mean temperature surfaces from the CARS temperature data. The data at each measurement plane are fitted to a cosine series bivariate function of order 5 (plane 1 only) or order 6 , with the number of fit parameters respectively 21 and 28. Fits are performed using commercial software (TableCURVE 3D, data available online at http://www.systat.com [cited 29 September 2003]). The number

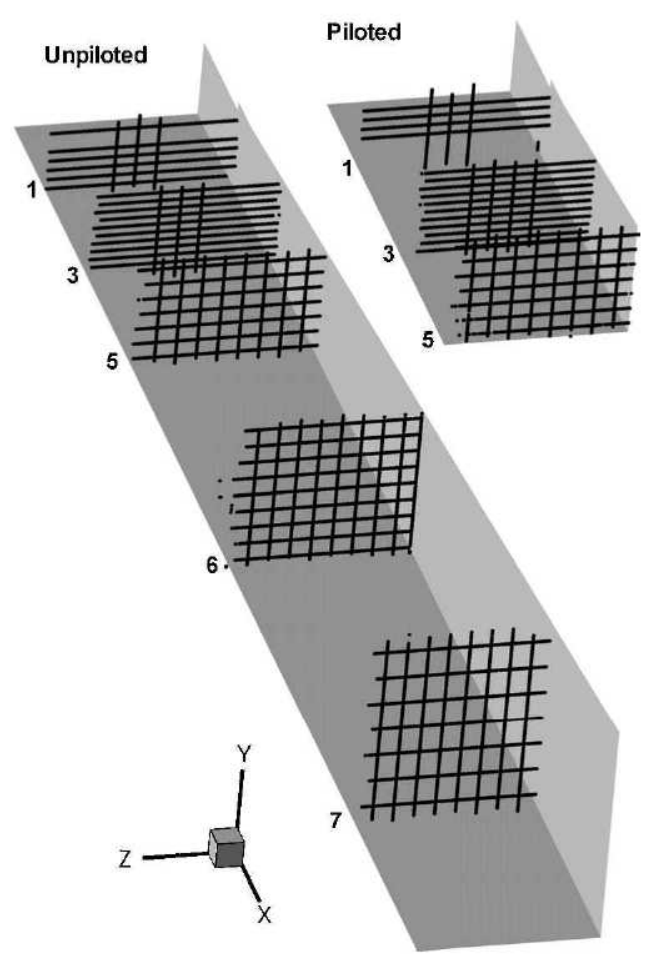

Fig. 5 Cutaway view of data acquisition points. 
of data points acquired per plane, $n$, is from 2000 to 4000 , and the standard deviation of the data from the fit at the various planes, $\sigma$, is from 196 to $304 \mathrm{~K}$. The uncertainty of the fitted functions, given by $1.96 \sigma \sqrt{ }(p / n)$ (Ref. 19), is between 36 and $59 \mathrm{~K}$ depending on the plane.Here, $p$ is the number of parameters of the fit. This uncertainty in the fits is actually an approximation representing the mean over all points to which the response model is fit. The true uncertainty depends on the specific location in a plane and is somewhat lower near the center of the plane and somewhat higher near the edge. Lower fit uncertainties could be achieved by increasing the number of data points acquired. However, this is not warranted given the previously described uncertainty in the CARS mean temperatures of $\pm 50 \mathrm{~K}$ due to fixed errors.

Note that if a more conventional approach had been adopted in which data were obtained at a few discrete spatial points, the number of samples required at each point to obtain an uncertainty of $\pm 50 \mathrm{~K}$ would have been approximately $(1.96 \times 304 \mathrm{~K} / 50 \mathrm{~K})^{2}=142$ samples/point. This number would allow only about ( 4000 samples/ plane $) /(142$ samples/point $)=28$ measurement points per plane. A functional representation of the surface temperature distribution over the measurement plane, with given uncertainty, provides better spatial coverage and a more compact representation than the 28 discrete points obtained with the same single-point uncertainty.

In addition to the estimates of mean temperature surfaces just described, surface estimates of the standard deviation of temperature, or root mean square temperature deviation, were obtained. The difference between the temperature and the mean obtained from the surface fit is obtained at each of the measurement points. These temperature deviations are squared and the resulting data are fit at each plane to a cosine series bivariate function of order 5 . Standard deviation of these fits is between 78,000 and $190,000 \mathrm{~K}^{2}$ and the fit uncertainty is between 15,000 and $32,000 \mathrm{~K}^{2}$. Typical values of uncertainty expressedas a percentageare $\pm 25,000 / 300^{2} \times 100= \pm 28 \%$. It should be emphasized that this uncertainty is caused by insufficient data points: as $n$ increases, the uncertainty becomes small.

Random deviations of temperature from the mean were traced to several sources. The deviation that we are interested in is inherent in the flow and is due to the fluid dynamical turbulent fluctuations. Another source is random error inherent in the CARS instrument. The standard deviation of this error was measured to be $\sim 100 \mathrm{~K}$. The uncontrolled systematic temperature variations, converted to random deviations by randomizing the order in which data were acquired at the various spatial locations, also contribute to the temperature fluctuations. The magnitude of these systematic variations is unknown but assumed to be small. Since these various sources of temperature deviation are statistically independent, the mean square deviations are additive. Thus, in addition to the large random error, the experimental fitted mean squared deviation surface is everywhere $\sim(100 \mathrm{~K})^{2}$ high. Given these high levels of uncertainty and error, the mean squared deviation surface fits are only qualitatively useful.

\section{Results}

\section{Surface Pressure and Temperature}

Surface pressure and temperature data are presented for two typical runs, one unpiloted and one piloted. These runs are chosen because the heater pressure and the gas flow rates to the heater, injector, and pilot are all very close to the respective averages over the total set of runs.

Surface pressure distributions for the pressure taps on the top and bottom walls on the centerline, averaged over a 1-s interval at $16 \mathrm{~s}$ into the run, are shown in Fig. 6. Uncertainty in these measurements varies widely, depending on location in the duct, but are typically $\sim \pm 3 \mathrm{kPa}$ due primarily to unsteadiness in the flow. Pressures vary widely in the upstream region due to the shock wave system created by the injectors and step. In the unpiloted case pressure generally falls, moving downstream due to divergence of the duct, until $0.4 \mathrm{~m}$, where it rises rapidly, peaking at $\sim 0.7 \mathrm{~m}$. Pressures for the piloted case are higher between the pilot $(x=0.074 \mathrm{~m})$ and $x=0.7 \mathrm{~m}$ due to combustion of gas from the pilot and main fuel injector in this upstream region. Downstream of $0.7 \mathrm{~m}$ the pressure drops smoothly

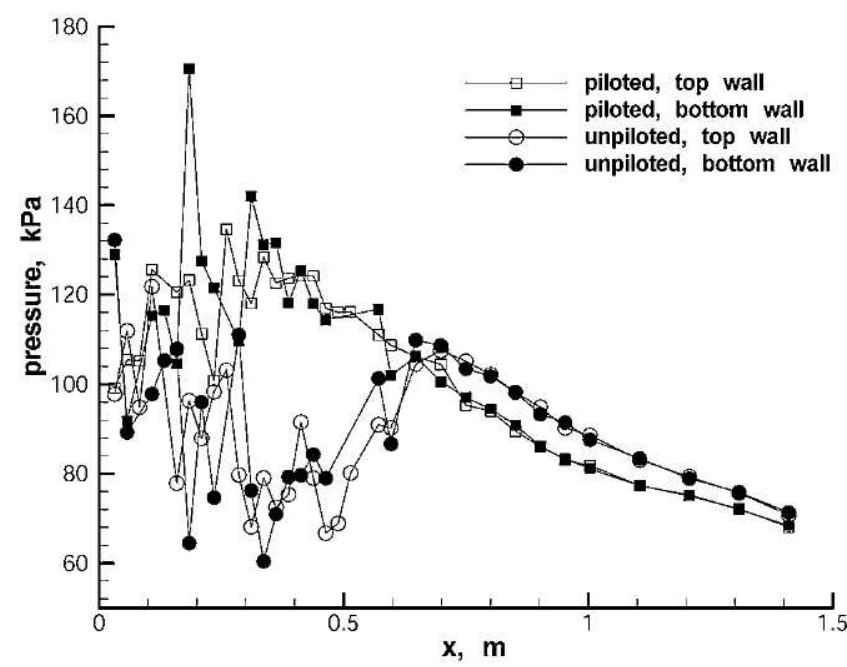

Fig. 6 Surface-pressure distributions along centerline of top and bottom walls.

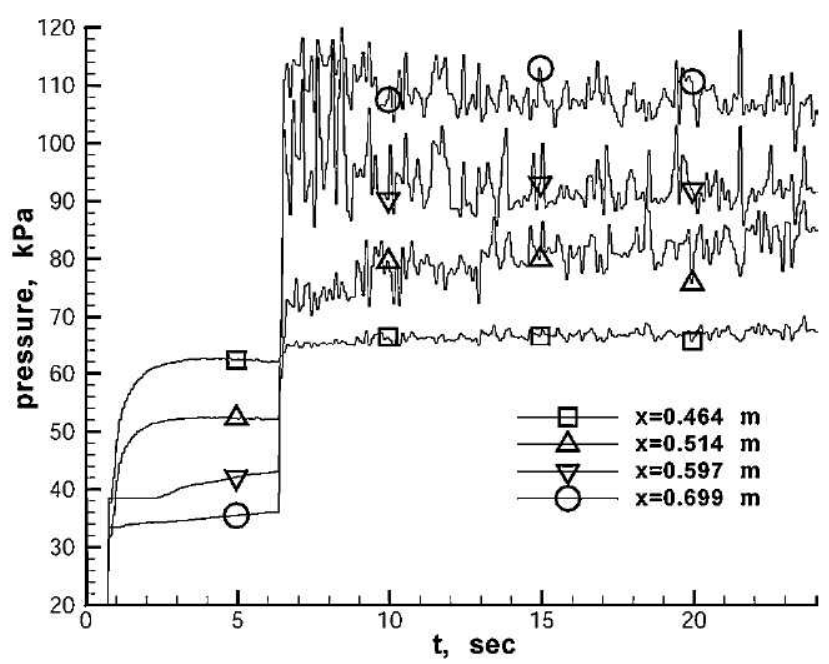

Fig. 7 Surface-pressure time histories at selected taps on top wall for the unpiloted case.

in both cases but is higher in the unpiloted case despite the lower total injected fuel rate. Differences between the two cases suggest that significant combustion of the fuel does not take place upstream of $0.4 \mathrm{~m}$ in the unpiloted case, but that the combustion of the fuel and air that are mixed then proceeds to completion by $0.7 \mathrm{~m}$. It believed that fuel and air are not fully mixed at $0.7 \mathrm{~m}$, so further mixing and combustion are expected to occur downstream.

Surface pressure time histories for the unpiloted case, on the top wall, for several pressure taps between 0.4 and $0.7 \mathrm{~m}$ (in the region of rapid pressure rise) are shown in Fig. 7. The heater flow is initiated at time $t_{0}=1 \mathrm{~s}$ and fuel injection commences at time $t_{1}=6.4 \mathrm{~s}$. It is seen that, after the initiation of heater flow, there are large fluctuations in the pressure but no significant trends. Thus, the location at which combustion takes place is not significantly affected by the increase in surface temperature that occurs during the course of a run. Much higher fluctuations in pressure are observed at taps downstream of the $x=0.464 \mathrm{~m}$ tap, suggesting that the combustion process is associated with a large increase in the level of local unsteadiness in the flow. The measured pressure is filtered by the response of the tubes between the surface and the transducer, and the response function of the transducer. Because the system time response is much longer than characteristic turbulence time scales of the flow, these data underestimate the unsteadiness.

Because the duct is uncooled, surface temperatures vary greatly during the course of the run, as seen in Fig. 8 for selected points in 


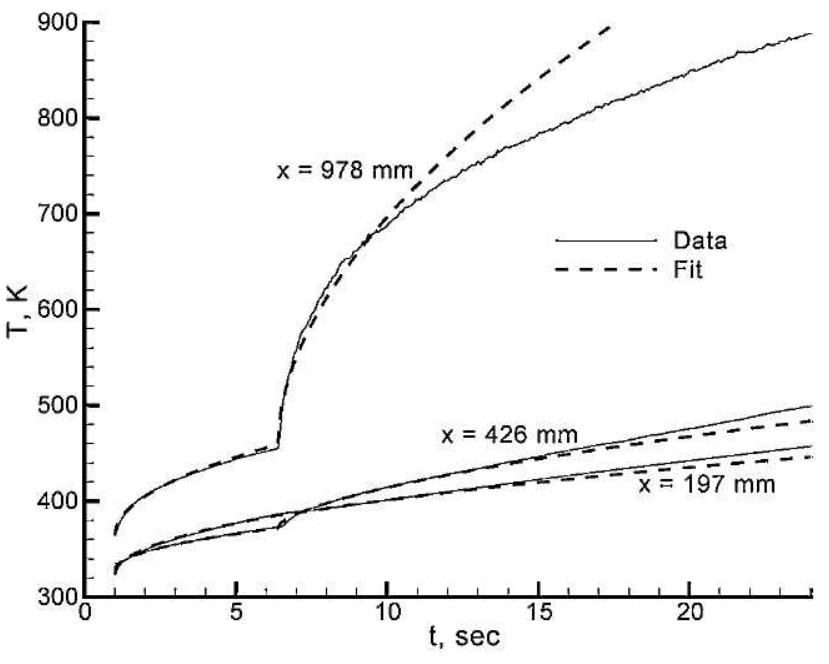

Fig. 8 Wall temperature history, unpiloted, top wall: data and fits.

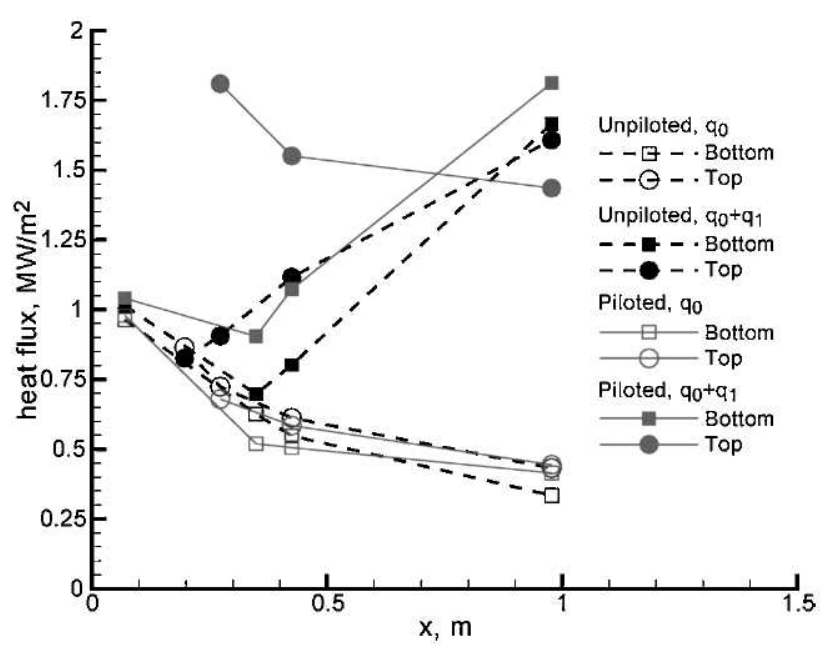

Fig. 9 Surface heat flux distributions: top and bottom walls, centerline.

the unpiloted case. In the copper section, temperature is typically $\sim 320 \mathrm{~K}$ at the start of fuel injection but rises to as high as $\sim 500 \mathrm{~K}$ in the unpiloted case and $\sim 590 \mathrm{~K}$ in the piloted case. In the carbonsteel section, temperature is typically $\sim 320 \mathrm{~K}$ at the start and as high as $\sim 890 \mathrm{~K}$ at the end. These variations are least-squares fit to the solution for wall temperature $T_{w}$ of a semi-infinite body at initially uniform temperature $T_{i}$ and subject to steps in surface heat flux from zero to $q_{0}$ at time $t_{0}$ and from $q_{0}$ to $q_{0}+q_{1}$ at $t_{1}$ (Ref. 27):

$$
\begin{gathered}
T_{w}-T_{i}=\frac{2 q_{0}}{k_{s}} \sqrt{\frac{\alpha_{s}\left(t-t_{0}\right)}{\pi}} \text { for } t_{0} \leq t<t_{1} \\
T_{w}-T_{i}=\frac{2 q_{0}}{k_{s}} \sqrt{\frac{\alpha_{s}\left(t-t_{0}\right)}{\pi}}+\frac{2 q_{1}}{k_{s}} \sqrt{\frac{\alpha_{s}\left(t-t_{1}\right)}{\pi}} \text { for } t \geq t_{1}
\end{gathered}
$$

The fit parameters are $T_{i}, q_{0}$, and $q_{1}$ and the fit is conducted out to $t=11 \mathrm{~s}$. As seen in Fig. 8, fits often diverge from the data beyond $11 \mathrm{~s}$, indicating that heat flux continues to change slowly during the run. The material property $\sqrt{ } \alpha_{s} / k_{s}$ is taken to be $36.7 \mathrm{~kW} \cdot \mathrm{K} / \mathrm{m}^{2} \mathrm{~s}^{1 / 2}$ for the copper duct and $12 \mathrm{~kW} \cdot \mathrm{K} / \mathrm{m}^{2} \mathrm{~s}^{1 / 2}$ for the steel duct. Heat fluxes are presented in Fig. 9, both top and bottom walls, on the centerline. Heat flux varies from point to point in the range 0.3 to $1.0 \mathrm{MW} / \mathrm{m}^{2}$ before injection $\left(q_{0}\right)$, and from 0.7 to $1.8 \mathrm{MW} / \mathrm{m}^{2}$ with injection $\left(q_{0}+q_{1}\right)$, where $q_{1}$ is the increase in heat flux. At a given point, $q_{0}$ varies 10 to $30 \%$ from run to run, probably reflecting variation in the initial temperature of the wall between runs. At downstream locations, $q_{1}$ is large due to injection and combustion.
In the piloted case, $q_{1}$ is large on the top wall, downstream of the main injector $(x=0.166 \mathrm{~m})$, indicating the presence of combustion products near the wall. In the unpilotedcase, $q_{1}$ is significant (though not so large as the piloted case) ahead of $0.7 \mathrm{~m}$ (where the pressure rise begins), consistent with incomplete combustion near the wall.

\section{CARS Temperatures}

Figures 10 and 11 are three-dimensional cutaway views of the duct showing contour plots of the fitted temperature functions and

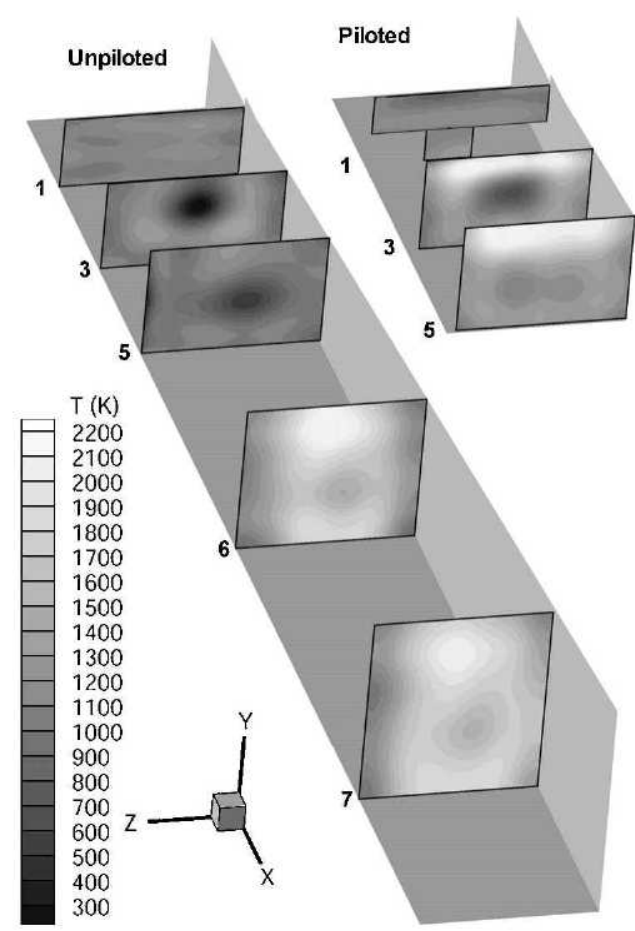

Fig. 10 Cutaway view of duct showing contour plots of mean temperature for unpiloted and piloted cases.

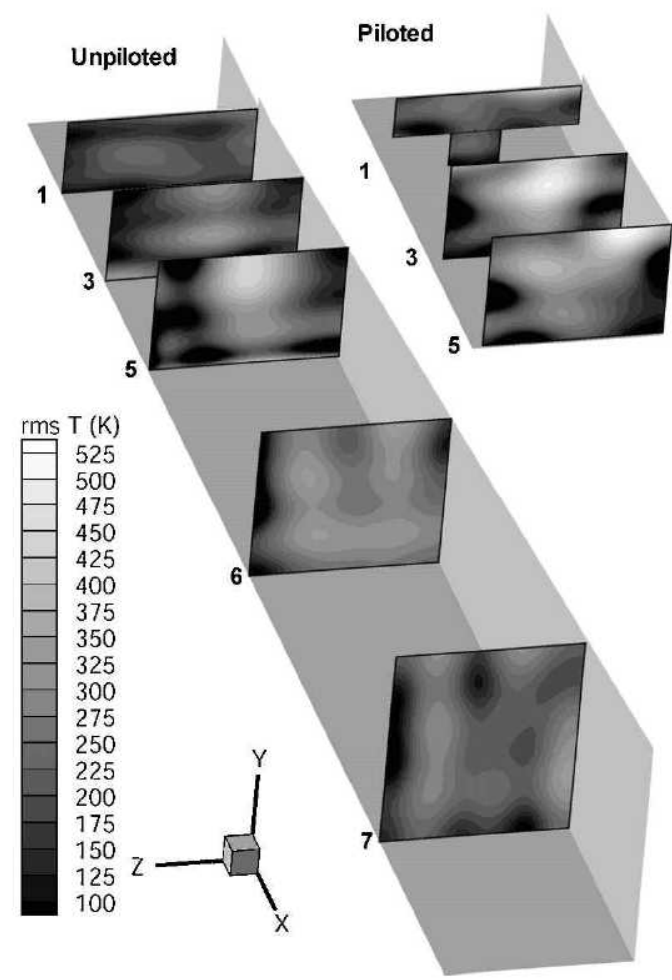

Fig. 11 Cutaway view of duct showing contour plots of rms temperature deviation for unpiloted and piloted cases. 
the square root of the fitted mean-square deviation functions (i.e., the rms deviation). Also shown are two of the four duct walls. Recall that the main fuel injector is on the top wall between planes 1 and 3 , and that the pilot injectors are on the top wall upstream of plane 1. An idea of the scale may be found by referring back to the dimensioned drawings in Fig. 2. Please note that the temperature measurements, although essentially instantaneous, are widely spaced in time relative to any characteristic time of the flow, and so consecutive measurements are uncorrelated. Thus, it was not possible to extract from these measurements such quantities as turbulence time scales.

Consider first the fitted mean temperature surfaces (Fig. 10). At plane 1 , in the unpiloted case, the temperature is fairly uniform, between 1030 and $1250 \mathrm{~K}$. The mean temperature of this plane is $1162 \mathrm{~K}$. This mean compares favorably with the value computed assuming one-dimensionalflow from the heater, which is $1187 \pm 60 \mathrm{~K}$. In the piloted case, the fitted temperature is also reasonably uniform, except that the temperature drops slightly close to the top wall, downstream of where the pilot fuel is injected. There is no indication of pilot fuel combustion in this plane. At plane 3 , in the unpiloted case, there is a region with temperature as low as $\sim 250 \mathrm{~K}$ at the center, which is the injected fuel plume. There is no evidence of combustion of the injected fuel. In the piloted case, there is a band of hot pilot fuel combustion products close to the top wall. The center of the main fuel plume may be seen as a cooler region with temperature as low as $\sim 650 \mathrm{~K}$, greater than in the piloted case, suggesting some combustion. Plane 5 is similar to plane 3 . Temperatures have risen near the center of the fuel plume, to as low as $\sim 550 \mathrm{~K}$ in the unpiloted case and $\sim 1250 \mathrm{~K}$ in the piloted case. The height of the region of hot combustion products near the top wall in the piloted case has increased. At planes 6 and 7, in the unpiloted case, temperatures have risen abruptly compared to plane 5 , suggesting nearly complete combustion (i.e., $\mathrm{H}_{2}$ and $\mathrm{O}_{2}$ do not coexist at any given point). In the regions in these planes with the highest temperatures $(\sim 2300 \mathrm{~K})$, the fuel and air are probably mixed to nearly stoichiometric proportions. The cooler region near the center $(\sim 1500 \mathrm{~K})$ is fuel rich. No significant quantities of injected fuel have penetrated to the sidewalls ( $\sim 1200$ to $1300 \mathrm{~K})$. Data are not acquired at planes 6 and 7 in the piloted case due to lack of time.

Figure 12 shows temperature measurements from a single vertical scan near the horizontal centerline for plane 5 in the unpiloted case. The temperature surface fit along this spatial line is also shown, with error bars, as are the mean temperatures from several fixed-point runs, also with error bars. The error bars of the surface fit are based on all the data in the plane and so include the effects of run-to-run variations. The error bars of the fixed points are based on the standard deviations of the given run, containing about 150 individual measurements. In neither case is uncertainty due to fixed errors included, which was given as $\pm 50 \mathrm{~K}$. It may be seen that the fixed-point mean temperatures agree with the surface

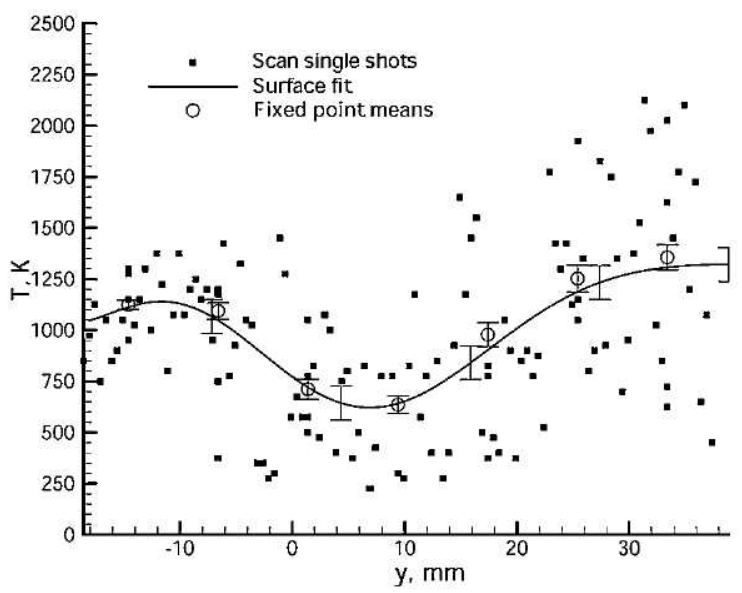

Fig. 12 Temperature data at $z=3.5 \mathrm{~mm}$, plane 5, unpiloted case. fit, providing confidence in the approach adopted and suggesting that run-to-run variation is not significant. This figure also illustrates graphically the relative levels of uncertainty and the high levels of fluctuations in the data. Note that the fluctuations either side of the temperature surface fit indicate real temperature fluctuations in the flow, because fluctuation levels are larger than the instrument precision error of $\sim 100 \mathrm{~K}$ (standard deviation). The presence of high temperature fluctuations should not be surprising since, at many points in the turbulent combusting flow, there will at certain times be hot combustion products and at other times cooler air or fuel.

Consider now the rms temperature deviation surfaces (Fig. 11). All the surfaces have irregular oscillations of amplitude $\sim 100 \mathrm{~K}$ due to random error. However, there are significant features present. At plane 1, for the unpiloted case, the rms deviation surface varies between $\sim 100$ and $\sim 230 \mathrm{~K}$. The standard deviations obtained from measurements at fixed points varied from 140 to $220 \mathrm{~K}$, significantly greater than that inherent in the instrument $(\sim 100 \mathrm{~K})$. Thus, there are significant real temperature fluctuations in the flow exiting the heater, with standarddeviation $\sim 100 \mathrm{~K}$ or greater. The rms deviation level in the piloted case is comparable, except that it rises close to the top surface, downstream of the pilots.

Several regions of large rms temperature deviation levels may be observed. One region of high rms deviation level, up to $\sim 440 \mathrm{~K}$, lies between the center of the fuel plume and the top wall in plane 5 of the unpiloted case. A second region, with rms deviation level up to $\sim 500 \mathrm{~K}$, lies near the fuel plume in plane 3 of the piloted case. In both cases, these regions appear to be ones where combustion is just beginning or is incomplete. This suggests that intermittency in degree of combustion is the main cause of the fluctuations; that is, in some lumps of fluid passing through the measurement plane combustion has taken place and in others it has not. The result for the unpiloted case is particularly interesting because there is little indication in the mean temperature plot of combustion having taken place, but solid evidence in the rms plot of intermittent combustion. One further region of large rms deviation level, with peak rms deviation level of $\sim 525 \mathrm{~K}$, lies on the right side near the top wall, in plane 5 , in the piloted case. The mean temperature is $\sim 2200 \mathrm{~K}$ at this location, much higher than for the other locations discussed, so the rms deviation level represented as a fraction of the mean is much lower than in these locations. It is probable that the temperature surface fit has greater error at the edge of the data plane. The high standard deviation at this location may simply be explained by greater error in the mean temperature surface.

The fitted mean temperature and rms temperature deviation surfaces, and the surface-pressureand heat flux data, provide a consistent description of the flowfield. In the unpiloted case, only small and intermittent combustion of the injected fuel is observed ahead of $x=0.4 \mathrm{~m}$. Downstream of $x=0.7 \mathrm{~m}$, combustion appears nearly complete. In the piloted case, combustion of the pilot fuel appears to take place between $x=0.122$ (at the step) and $0.274 \mathrm{~m}$. There also appears to be significant combustion of the main injected fuel by $x=0.274 \mathrm{~m}$. These results are not consistent with CFD calculations performed prior to the commencement of the experimental work, ${ }^{3}$ which predicted combustion in the vicinity of injection in both unpiloted and piloted cases. Consequently, this experiment provides a test case for CFD that is more challengingthan anticipated. Accurate calculation will require accurate modeling of both the chemical kinetics and the turbulence-chemistry interactions, as well as accurate modeling of the turbulent mixing.

\section{Conclusions}

CARS thermometry and MDOE techniques have been successfully applied to a simple supersonic combustor. Functions that provide an estimate of the mean temperature and rms temperature deviation surfaces at various planes in the flow have been obtained. Additional data include surface pressure and temperature distributions. Boundary conditions and uncertaintiesare well characterized, and the data are useful for the validation of CFD codes used in the design of supersonic combustors. Accurate calculation of the aggregate data set will require accurate modeling of both the chemical 
kinetics and the turbulence-chemistry interactions, as well as accurate modeling of the turbulent mixing.

\section{Acknowledgments}

The support of the NASA Langley Research Center through Grant NCC1-370 and contributions by J. P. Drummond, M. W. Smith, and G. S. Diskin are gratefully acknowledged.

\section{References}

${ }^{1}$ Cutler, A. D., Danehy, P. M., Springer, R. R., DeLoach, R., and Capriotti, D. P., "CARS Thermometry in a Supersonic Combustor for CFD Code Validation," AIAA Paper 2002-0743, Jan. 2002.

${ }^{2}$ Danehy, P. M., DeLoach, R., and Cutler, A. D., "Application of Modern Design of Experiments to CARS Thermometry in a Model Scramjet Engine," AIAA Paper 2002-2914, June 2002.

${ }^{3}$ Drummond, J. P., Diskin, G. S., Cutler, A. D., and Danehy, P. M., "FuelAir Mixing and Combustion in Scramjets," AIAA Paper 2002-3878, July 2002.

${ }^{4}$ Cutler, A. D., Diskin, G. S., Danehy, P. M., and Drummond, J. P., "Fundamental Mixing and Combustion Experiments for Propelled Hypersonic Flight," AIAA Paper 2002-3879, July 2002.

${ }^{5}$ Cutler, A. D., and White, J. A., "An Experimental and CFD Study of a Supersonic Coaxial Jet," AIAA Paper 2001-0143, Jan. 2001.

${ }^{6}$ Cutler, A. D., Carty, A. A., Doerner, S. E., Diskin, G. S., and Drummond, J. P., "Supersonic Coaxial Jet Flow Experiment for CFD Code Validation," AIAA Paper 99-3588, June 1999.

${ }^{7}$ Springer, R. R., Cutler, A. D., Diskin, G. S., and Smith, M. W., "Conventional/Laser Diagnostics to Assess Flow Quality in a Combustion-Heated Facility," AIAA Paper 99-2170, June 1999.

${ }^{8}$ Eckbreth, A. C., Laser Diagnostics for Combustion Temperature and Species, 2nd ed., Combustion Science and Technology Series, Gordon and Breach, New York, 1996.

${ }^{9}$ Smith, M. W., Jarrett, O., Jr., Antcliff, R. R., Northam, G. B., Cutler, A. D., and Taylor, D. J., "Coherent Anti-Stokes Raman Spectroscopy Temperature Measurements in a Hydrogen-Fueled Supersonic Combustor," Journal of Propulsion and Power, Vol. 9, No. 2, 1993, pp. 163-168.

${ }^{10}$ Meier, W., Barlow, R. S., Chen, Y.-L., and Chen, J.-Y, "Raman/ Rayleigh/LIF Measurements in a Turbulent $\mathrm{CH}_{4} / \mathrm{H}_{2} / \mathrm{N}_{2}$ Jet Diffusion Flame: Experimental Techniques and Turbulence-Chemistry Interaction" Combustion and Flame, Vol. 123, 2000, p. 326-343.

${ }^{11}$ McMillin, B. K., Palmer, J. L., and Hanson, R. K., "Temporally Resolved, 2-Line Fluorescence Imaging of NO Temperature in a Transverse Jet in a Supersonic Cross-Flow," Applied Optics, Vol. 32, No. 36, 1993, pp. 7532-7545.

${ }^{12}$ Donohue, J. M., and McDaniel, J. C., "Complete Three-Dimensional
Multiparameter Mapping of a Supersonic Ramp Fuel Injector Flowfield," AIAA Journal, Vol. 34, No. 3, 1996, pp. 455-462.

${ }^{13}$ Forkey, J. N., Lempert, W. R., and Miles, R. B., "Accuracy Limits for Planar Measurements of Flow Field Velocity, Temperature and Pressure Using Filtered Rayleigh Scattering," Experiments in Fluids, Vol. 24, No. 2, 1998, pp. 151-162.

${ }^{14}$ Jonuscheit, J., Thumann, A., Schenk, M., Seeger, T., and Leipertz, A., "One-Dimensional Vibrational Coherent Anti-Stokes RamanScattering Thermometry," Optics Letters, Vol. 21, No. 19, 1996, pp. 1532-1534.

${ }^{15}$ Box, G. E. P., Hunter, W. G., and Hunter, J. S., Statistics for Experimenters. An Introduction to Design, Data Analysis, and Model Building, Wiley, New York, 1978.

${ }^{16}$ Cochran, W. G., and Cox, G. M., Experimental Designs, 2nd ed., Wiley Classics Library ed., Wiley, New York, 1992.

${ }^{17}$ Montgomery,D. C., Design and Analysis of Experiments, 5th ed., Wiley, New York, 2001.

${ }^{18}$ Fisher, R. A., The Design of Experiments, 8th ed., Oliver and Boyd, Edinburgh, 1966.

${ }^{19}$ Box, G. E. P., and Draper, N. R., Empirical Model Building and Response Surfaces, Wiley, New York, 1987.

${ }^{20}$ Myers, R. H., and Montgomery, D. C., Response Surface Methodology: Process and Product Optimization Using Designed Experiments, Wiley, New York, 1995.

${ }^{21}$ Draper, N. R., and Smith, H., Applied Regression Analysis, 3rd ed., Wiley, New York, 1998.

${ }^{22}$ Montgomery, D. C., and Peck, E. A., Introduction to Linear Regression Analysis, 2nd ed., Wiley, New York, 1992.

${ }^{23}$ Antcliff, R. R., Hillard, M. E., and Jarrett, O., Jr., "Intensified Silicon Photodiode Array Detector Linearity: Application to Coherent AntiStokes Raman Spectroscopy," Applied Optics, Vol. 23, No. 14, 1984, pp. 2369-2375.

${ }^{24}$ Eckbreth, A. C., "Optical Splitter for Dynamic Range Enhancement of Optical Multichannel Detectors," Applied Optics, Vol. 22, July 1983 , pp. 2118-2123.

${ }^{25}$ Grisch, F., Bouchardy, P., Pealat, M., Chanetz, B., Pot, T., and Coet, M. C., "Rotational Temperature and Density-Measurements in a Hypersonic Flow by Dual-Line Cars," Applied Physics B-Photophysics and Laser Chemistry, Vol. 56, No. 1, 1993, pp. 14-20.

${ }^{26}$ Clark, G., and Farrow, R. L., The CARSFT Code: User and Programmer Information, Sandia National Laboratories, Livermore, CA, 1990.

${ }^{27}$ Kreith, F., and Bohn, M. S., Principles of Heat Transfer, 6th ed., Brooks/Cole, Pacific Grove, CA, 2001, Sec. 2.6.3.

R. P. Lucht Associate Editor 